\title{
Fractal Surface Structure of Food Materials Recognized by Different Molecules
}

\author{
Toru Suzuki and Toshimasa YANO* \\ Department of Food Technology and Engineering, Tokyo University of Fisheries, \\ Konan 4, Minato-ku, Tokyo I08, Japan. \\ * Department of Agricultural Chemistry, Faculty of Agriculture. The University of Tokyo, \\ Yayoi 1, Bunkyo-ku, Tokyo 113, Japan \\ Received August 24, 1990
}

\begin{abstract}
The moles of monolayer adsorption of defatted rice flour, wheat flour, and corn fiber, and of active carbon as a reference, were measured using argon, nitrogen, krypton, ethane, propane, and butane. The relation between the moles of monolayer adsorption and the cross-sectional area of adsorbent molecules was not as expected for the smooth surface, but showed a fractal surface structure. A fractal dimension, $D_{\sigma}$, was calculated from the logarithmic relation between the moles of monolayer adsorption and the cross-sectional area of adsorbent molecules. The values of $D_{\sigma}$ were not equal in general to the values of another fractal dimension, $D_{s}$, calculated in our preceding paper from the logarithmic relation between the specific surface area and the particle diameter.
\end{abstract}

The fractal concept proposed by Mandelbrot $^{13}$ in 1975 seems to be useful for quantitative understanding of the irregular structure of foods. Pfeifer et al. ${ }^{2,3)}$ showed that for active carbon, or silica gel, the relation between the specific surface area and particle diameter was not as commonly believed, but as the following:

$$
S_{w} \propto n_{m} \propto d_{p}^{D_{s}-3}
$$

where $d_{p}$ is the particle diameter, $D_{s}$ a fractal dimension, $n_{m}$ the monolayer moles of a gas adsorbed on a gram of solids, and $S_{w}$ the specific surface area recognized by the gas molecule adsorbed on the solid. They also showed that the value of $n_{m}$, which is reversely proportional to the cross-sectional area of molecules for the ideally smooth surface, was not as that for the smooth surface but as the following:

$$
n_{m} \propto \sigma^{-D_{\sigma} / 2}
$$

where $D_{\sigma}$ is another fractal dimension, and $\sigma$ the cross-sectional area of the adsorbent molecules. In Eqs. (1) and (2), the values of $D_{s}$ and $D_{\sigma}$ may take any non-integral real number between 2 and 3 . Though Pfiefer et al. ${ }^{4)}$ assumed that $D_{s}$ is equal to $D_{\sigma}$, it may not to be so in general.

If $D_{s}$ is independent of $D_{\sigma}$, the combination of Eqs. (1) and (2) gives

$$
n_{m} \propto d_{p}^{D_{s}-3} \cdot \sigma^{-D_{\sigma} / 2}
$$

Since $S_{w} \propto n_{m}-\sigma$, we obtain the following equation from Eq. (3)

$$
S_{w} \propto d_{p}^{D_{s}-3} \cdot \sigma^{\left(2-D_{n}\right) / 2}
$$

Equation (4) suggests that the specific area of a material may be differently recognized by molecules of different size. In our preceding paper $^{5)}$ we found that the surface porous structures of rice flour, wheat flour, and corn fiber, before and after defatting, and milk powders, were fractal and their irregularity was evaluated by the fractal dimension $D_{s}$. We also showed that the specific surface areas measured using $\mathrm{N}_{2}$ and $\mathrm{Kr}$ were different. Although this is against common sense, in which the surface is implicitly assumed to be smooth, it is acceptable, from the fractal viewpoint, for an irregular surface looked at with molecular-level scales.

As for whether $D_{s}$ is equal to $D_{\sigma}$, Nagai and Yano ${ }^{6)}$ showed that the values of $D_{s}$ and $D_{\sigma}$ 
were not equal for dried starch gels.

The purpose of this paper is to study more (1) on the different recognition of the specific surface area by different kinds of molecules, and (2) on whether $D_{s}$ is equal to $D_{\sigma}$, using the same materials used in our preceding paper. ${ }^{\text {) }}$

\section{Materials and Methods}

Materials. Rice flour, wheat flour, and corn fiber, before and after defatting, were used. The sample materials were the same as those used in our preceding paper, ${ }^{57}$ although the mean particle diameter was $26 \mu \mathrm{m}$ for the rice flour and wheat flour, and $45 \mu \mathrm{m}$ and $71 \mu \mathrm{m}$ for the corn fiber. Commercial active carbon was also used as a reference.

Moles of monolayer adsorption: using argon, nitrogen, ethane, krypton, propane, and butane, the adsorption isotherms were measured with an Accusorb 2100 (Micromeritics Co.) at temperatures shown in Table I after the sample was completely dried at $40^{\circ} \mathrm{C}$ under reduced pressure. The moles of monolayer adsorption were calculated from the adsorption isotherms using the following B.E.T. plot:

$$
\frac{P / P_{s}}{V\left(1-P / P_{s}\right)}=\frac{1}{V m C}+\frac{(C-1)\left(P / P_{s}\right)}{V m C}
$$

where $C[-]$ is constant, $P[\mathrm{mmHg}]$ an adsorption equilibrium pressure of a gas, $P_{s}[\mathrm{mmHg}]$ the saturation pressure of the gas at the given temperature, $V[\mathrm{ml} / \mathrm{g}]$ an adsorbed volume of the gas per unit weight of solid at the adsorption equilibrium at the standard temperature and pressure (STP), and $V m[\mathrm{ml} / \mathrm{g}]$ the value of $V$ for monolayer coverage. The moles of monolayer adsorption $n_{m}[\mathrm{~mol} / \mathrm{g}]$ were calculated to be

$$
n_{m}=V m / 22400
$$

The purity of the argon and the nitrogen gas used was guaranteed to be $99.9995 \%$ that of the other was $99.9 \%$.

Table I. Molecular Cross-sectional Area AND EXPERIMENTAL CONDITIONS

\begin{tabular}{lccc}
\hline Gas & $\begin{array}{c}\text { Molecular } \\
\text { cross- } \\
\text { sectional } \\
\text { area } \\
\left(\mathrm{nm}^{2}\right)\end{array}$ & $\begin{array}{c}\text { Temp. } \\
\left({ }^{\circ} \mathrm{C}\right)\end{array}$ & $\begin{array}{c}\text { Saturated } \\
\text { pressure } \\
(\mathrm{mmHg})\end{array}$ \\
\hline $\mathrm{Ar}$ & 0.147 & -196 & 213 \\
$\mathrm{~N}_{2}$ & 0.162 & -196 & 760 \\
$\mathrm{Kr}$ & 0.202 & -196 & 3.5 \\
Ethane & 0.230 & -78 & 1303 \\
Propane & 0.364 & -78 & 111 \\
Butane & 0.448 & 0 & 774 \\
\hline
\end{tabular}

Table I lists the molecular cross-sectional area of the gases and the saturation gas pressure calculated from the Antoin equation ${ }^{7)}$ at the temperature of measurement.

\section{Results}

Since lipid-containing raw samples absorbed much ethane, propane, and butane, the B.E.T. plots did not give straight lines. Therefore, the monolayer adsorption was measured only for defatted materials.

Figure 1 shows the adsorption isotherms of six gases on the defatted wheat flour. The relative pressure on the abscissa is an equilibrium pressure of a gas divided by the saturation pressure of the gas shown in Table I, and STP on the ordinate means standard temperature and pressure. The smaller the molecule, the larger the adsorption volume. Figures 2 and 3 show the adsorption isotherms of the various gases on the defatted rice flour and defatted corn fiber $(45 \mu \mathrm{m}$ in the mean particle diameter) in low relative pressure, less than about 0.35 , since it was enough to calculate the monolayer value. Though the results for the defatted corn fiber of $71 \mu \mathrm{m}$ in mean particle diameter are not shown here, the adsorption isotherms were similar to those shown in Figs. 2 and 3. As Figs. 1 through 3

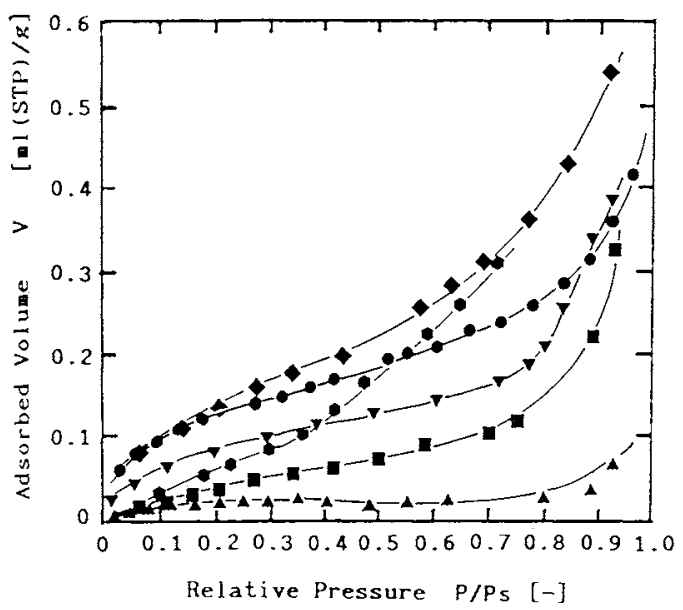

Fig. 1. Adsorption Isotherms of Various Gases on Defatted Wheat Flour (mean particle diameter $26 \mu \mathrm{m}$ ).

$(\bullet)$, argon; ( $)$, nitrogen; ( $)$, krypton; ( $)$, ethane; $(\mathbf{\square})$, propane; (A), butane. 


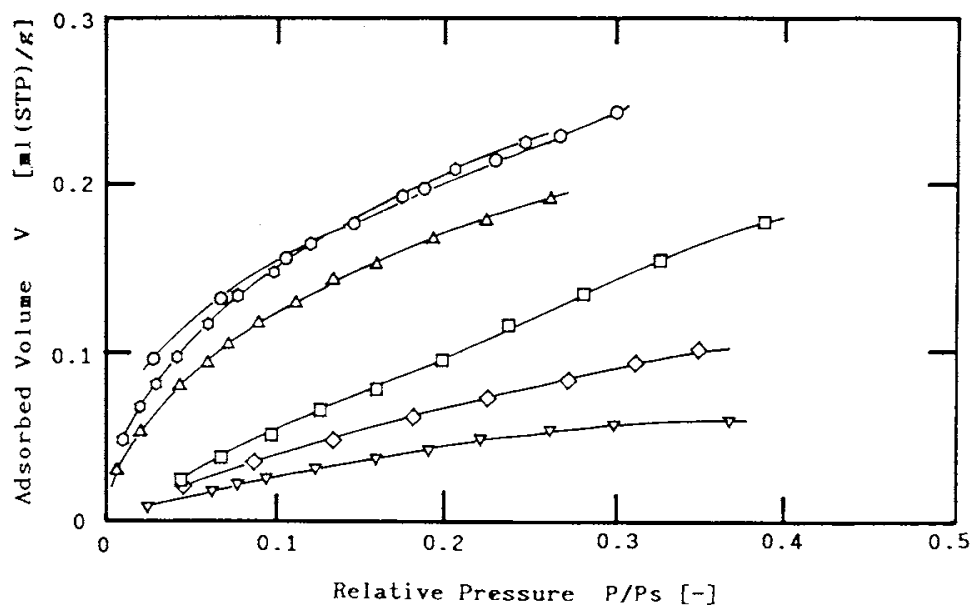

Fig. 2. Adsorption Isotherms of Various Gases on Defatted Rice Flour (mean particle diameter $26 \mu \mathrm{m}$ ). $(\circlearrowleft)$, argon; $(\bigcirc)$, nitrogen; $(\triangle)$, krypton; $(\square)$, ethane; $(\diamond)$, propane; $(\nabla)$, butane.

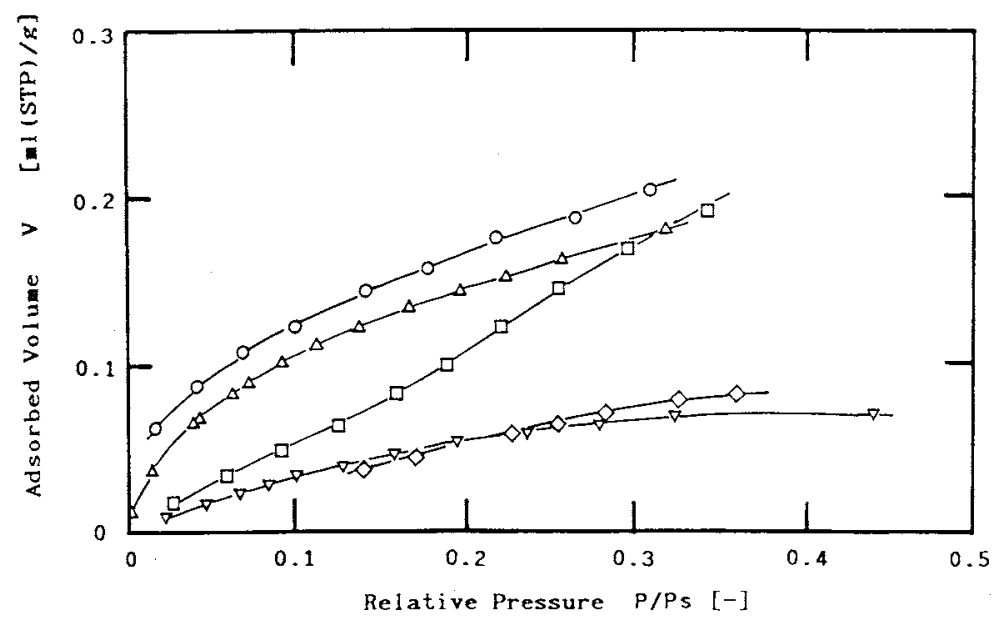

Fig. 3. Adsorption Isotherms of Various Gases on Defatted Corn Fiber (mean particle diameter $45 \mu \mathrm{m}$ ). $(\bigcirc)$, nitrogen; $(\triangle)$, krypton; $(\square)$, ethane; $(\diamond)$, propane; $(\nabla)$, butane.

show, the adsorbed volume decreased as the adsorbent molecular size increased, but the point of interest is the dependence of the monolayer moles $\left(n_{m}\right)$ of the gases on the cross-sectional areas $(\sigma)$ of the gases.

Figure 4 shows sample B.E.T. plots of propane gas for three samples. Good linear relations were obtained. The value of $V_{m}$ was obtained from the intersection of the ordinate and the slope of the lines according to Eq. (5), from which $n_{m}$ was calculated by Eq. (6).

Figure 5 shows the relation between monolayer values $\left(n_{m}\right)$ and molecular cross- sectional area $(\sigma)$. Linear relations of $\log n_{m} v s$. $\log \sigma$ were obtained. Fitting these data to Eq. (2), the fractal dimension $D_{\sigma}$ for the defatted materials and active carbon was calculated as shown in Table II. For the defatted corn fibers of $45 \mu \mathrm{m}$ and $71 \mu \mathrm{m}$ in particle diameter, the value of $n_{m}$ was higher for the former than the latter as shown in Fig. 5, but the fractal dimension $D_{\sigma}$ was not very different. In Table II, the value of $D_{s}$ found in our preceding paper $^{5)}$ using the same samples as used in this paper is shown for comparison. 


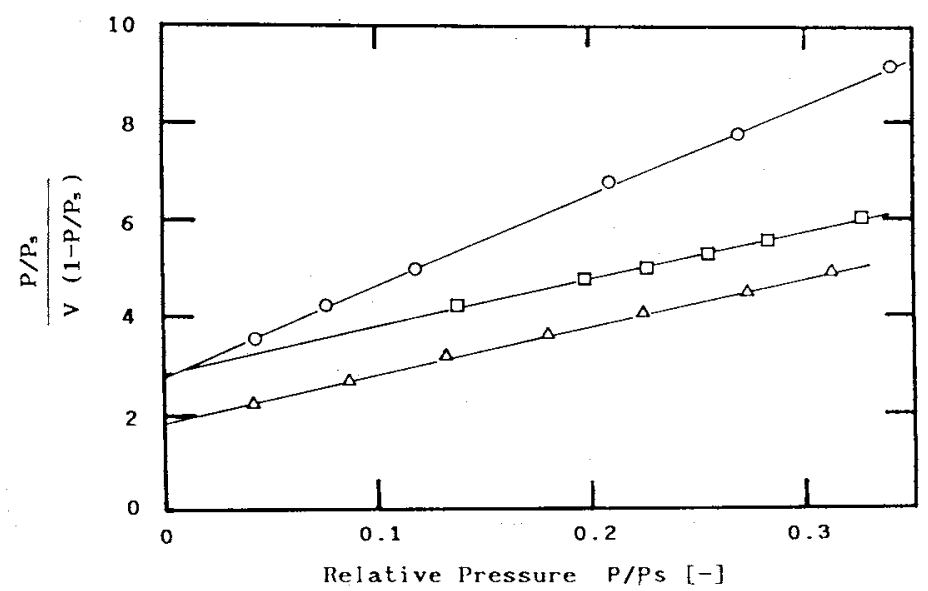

Fig. 4. B.E.T. Plot to Calculate Monolayer Values of Propane Gas.

Defatted wheat flour, $(O)$; defatted rice fiour $(\triangle)$; defatted corn fiber, $(\square)$.

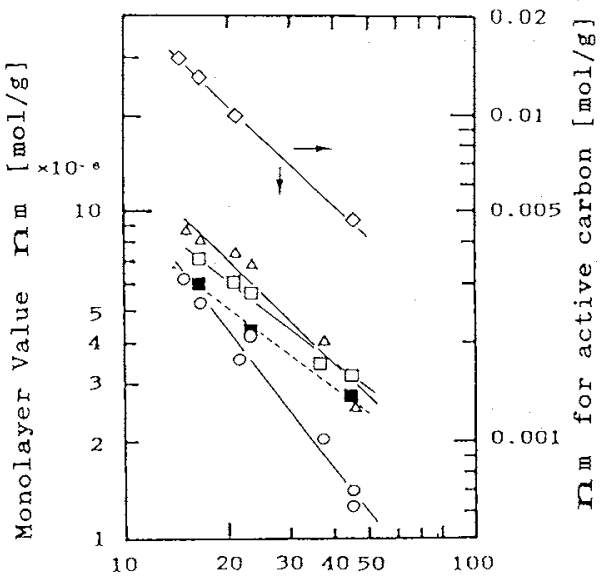

Cross-sectional Area of Molecules $\sigma\left[\AA^{2}\right]$

Fig. 5. The Relation between Monolayer Values $n_{m}$ and Cross-sectional Area $\sigma$ of Molecules.

Defatted wheat flour, $(\bigcirc)$; defatted rice flour $(\triangle)$; defatted corn fiber, ( $\square$ ) for $45 \mu \mathrm{m}$ and ( $\mathbf{n}$ ) for $71 \mu \mathrm{m}$; and active carbon, $(\diamond)$.

Table II. Comparison Between $D_{s}$ And $D_{\sigma}$ oF THE SAME MATERIals

\begin{tabular}{ccc}
\hline Sample & $D_{s}$ & $D_{\sigma}$ \\
\hline Defatted rice flour & 2.29 & 2.10 \\
Defatted wheat flour & 2.55 & 2.67 \\
Defatted corn fiber & & \\
$45 \mu \mathrm{m}$ & 2.61 & 1.59 \\
$71 \mu \mathrm{m}$ & 2.61 & 1.50 \\
Active carbon & - & 2.10
\end{tabular}

\section{Discussion}

Judging from the value of $D_{\sigma}$, the surfaces of defatted rice flour and active carbon used in this research were relatively smooth, while the surface of defatted wheat flour was irregular and fractal in nature. That is, the specific surface area of the defatted wheat flour was differently recognized by different size molecules. Pfeifer et $a{ }^{3}{ }^{3)}$ showed that fractal dimension $D_{\sigma}$ of active carbon changed from 3.03 to 1.94 with the progress of the degree of activation. Therefore, the active carbon used in this report was probably a highly activated one.

The value of $D_{\sigma}$ is usually considered ${ }^{2)}$ to be between 2.0 and 3.0. However, $D_{\sigma}$ for the defatted corn fibers were 1.59 and 1.50. This might happen for two reasons. One is the effect of some remaining lipid that absorbed hydrocarbon gases, making the "monolayer" adsorption incorrect. The defatting of the materials used was not complete, as shown in our preceding paper ${ }^{5)}$ by chemical analysis. And, even if the defatting be complete, it would not be certain that the gases were adsorbed only on the "surface" of a fibrous material. The surface structure of biological materials to be measured has an uncertainty in its definition. The other reason may be the effect of molecular shape. A long molecule might not go into a 
hole which a sphere of the same cross-sectional area could go into. On the other hand, a long molecule might be packed more densely than the sphere on the surface. The constraint $D_{\sigma}=2-3$ may have to be examined more carefully.

Comparison of $D_{s}$ obtained in our preceding paper ${ }^{5 t}$ with $D_{\sigma}$ obtained in this paper (see Table II) shows that, although the differences between $D_{s}$ and $D_{\sigma}$ were not significant for the defatted rice flour and defatted wheat flour, the value of $D_{s}$ in general may not be equal to $D_{\sigma}$ as shown for the defatted corn fiber. This suggests that $D_{\sigma}$ does not necessarily evaluate a simple surface structure as previousy stated for corn fiber. The inequality between $D_{s}$ and $D_{\sigma}$ was also observed by Nagai and Yano $^{6)}$ for xero-gels of starch. The assumption $D_{s}=D_{\sigma}$ adopted by Pfeifer et $a l .{ }^{4)}$ may not be applicable in general.

Since the values of $D_{s}$ and $D_{\sigma}$ were estimated as shown in Table II, the monolayer moles of a gas adsorbed on a gram of sample solids, $n_{m}$, and so the specific surface area recognized by the gaseous molecule, $S_{w}$, were predicted as a whole by Eqs. (3) and (4), respectively, as a function of $d_{p}$ and $\sigma$.

The fractal analyses made in our preceding paper $^{5)}$ and in this paper show a way for the quantitative evaluation of irregular structure of food materials, which may be used in food processings for improvements of adsorption selectivity, mass transfer characteristics including surface diffusion, reaction characteristics on solids, and physical properties including texture.

Acknowledgments. The authors express their thanks for the financial supports by the grant-in-aid for scientific research from the Ministry of Education, Science and Culture of Japan, and the Food Science Institute Foundation, Japan (Ryoshoku-Kenkyukai).

\section{References}

1) B. B. Mandelbrot, "The Fractal Geometry of Nature," Freeman, San Francisco, 1984.

2) P. Pfeifer and D. Avnir, J. Chem. Phys., 79, 3558 (1983).

3) D. Avnir, D. Farin and P. Pfeifer, J. Chem. Phys., 79, 3566 (1983).

4) P. Pfeifer, D. Avnir and D. Farin, J. Stat. Phys., 36, 699 (1984).

5) T. Suzuki and T. Yano, Agric. Biol. Chem., 54, 3131 (1990).

6) T. Nagai and T. Yano, J. Food Sci., 55, 1334 (1990).

7) R. C. Reid, J. M. Prausnitz and T. K. Sherwood, "The Properties of Gases and Liquids," 3rd Ed., MacGraw-Hill, 1977.

8) A. L. McClellan and H. F. Harnsberger, J. Colloid and Interface Sci., 23, 577 (1967). 